\section{Combination Immunotherapies}

\section{INTEGRATIVE IMMUNOMICS HIGHLIGHT THE IMMUNOMODULATORY IMPACT OF NEOADJUVANT CHEMOTHERAPY AND IMMUNE-BASED TREATMENTS IN RESECTED NON-SMALL-CELL LUNG CANCER}

Stephanie Schmidt*, Younghee Lee, Cheuk Leung, Lorenzo Federico, Heather Lin, Annikka Weissferdt, Apar Pataer, Hitoshi Dejima, Alejandro Francisco-Cruz, Frank Rojas, Luisa Solis, Edwin Parra, Monika Pradhan, Haiping Guo, William William, Alexandre Reuben, Humam Kadara, Ignacio Wistuba, Jianjun Zhang, Stephen Swisher, Ara Vaporciyan, Marcelo Negrao, Christopher Bristow, Timothy Heffernan, Chantale Bernatchez, Jack Lee, John Heymach, Boris Sepesi, Don Gibbons, Cara Haymaker, Tina Cascone. The University of Texas MD Anderson Cancer Center, Houston, TX, United States

Background How neoadjuvant chemo-immunotherapy modulates tumor immune composition and response is not completely understood. We interrogate immunomodulation of neoadjuvant platinum-based chemotherapy $(\mathrm{C})$, nivolumab $(\mathrm{N})$, and N-plus-C (NC) and their connections to therapeutic efficacy in resected non-small cell lung cancer (NSCLC) by integrating immunomic data from the ImmunogenomiC PrOfiling of NSCLC (ICON) study and NEOSTAR trial cohorts.

Methods In NEOSTAR (NCT03158129), patients with stage IIIIA (single N2) resectable NSCLC (AJCC7th) received N (3 $\mathrm{mg} / \mathrm{kg}$ IV, D1,15,29); patients with stage IB( $\geq 4 \mathrm{~cm}$ )-IIIA (single N2) resectable NSCLC received NC (N $360 \mathrm{mg}$ IV plus C, D1,22,43 for 3 cycles, every 3 weeks) before surgery; major pathologic response (MPR) was the primary endpoint. In ICON, patients with stage $\mathrm{IB}(\geq 4 \mathrm{~cm})$-IIIA resectable NSCLC received $C$ before surgery. Surgically resected tumor samples underwent immune profiling via flow cytometry $(n=16,13,9$ for C,N,NC), immunohistochemistry (IHC;n=0,18,14), and multiplexed immunofluorescence $(\mathrm{mIF} ; \mathrm{n}=28,16,10)$. Treatmentassociated immunomodulation and associations with therapeutic efficacy were analyzed using: 1) a shared nearest neighbors-based network we developed linking measurements across datasets; 2) MetaCyto, a specialized cytometry analysis method for identifying cell subsets by clustering.

Results We holistically explored the immunomic data by integration across cohorts. Through hierarchical regression of the integrated data, we determined the overall effect of a given treatment controlling for the presence or absence of the other treatment.

We examined C's effects across all cohorts controlling for $\mathrm{N}$. Across all patients, regardless of MPR, C is associated with immunosuppression, increasing PD1+ $\mathrm{T}$ cell $(\mathrm{CD} 45+\mathrm{CD} 3+)$ populations: regulatory (CD4+CD25+FOXP3+), helper (CD4 + ), and effector (CD8+) (effect size(ES):1.48,1.61,1.26; $\mathrm{q}<0.05)$. C also decreases proliferative $(\mathrm{Ki} 67+)$ populations: helper and effector T cells as well as NK (CD45+CD3-CD56 $+)$ cells (ES:-1.27,-1.43;-1.36; $\mathrm{q}<0.05)$. In patients without MPR (i.e., non-responding patients), immunosuppression appears heightened by increased $\mathrm{Ki67}+$ regulatory $\mathrm{T}$ cells (ES:1.86;q<0.05).

Conversely, we examined N's effects across all cohorts controlling for C. Across all patients, regardless of MPR, $\mathrm{N}$ is associated with immune activation, increasing ICOS $+\mathrm{T}$ cell populations: regulatory, helper, and effector (ES:1.29,1.29,1.47; $\mathrm{q}<0.05)$. Comparing $\mathrm{N}$ and $\mathrm{NC}$ reveals that adding $\mathrm{C}$ may drive exhaustion by increasing TIM3+ regulatory, helper and effector $\mathrm{T}$ cells (ES:1.16,1.17,1.23; $\mathrm{q}<0.05)$, an effect more pronounced in non-responding patients (ES:1.31,1.33,1.35; $\mathrm{q}<0.05)$.

Conclusions We report the first integrated examination of the immunomodulatory effect of neoadjuvant C and N. C is associated with immunosuppression while $\mathrm{N}$ with immune activation; together, $\mathrm{N}$ appears to lessen C's suppressive effects. Incorporation of transcriptomics into this integrated network of flow cytometry, mIF, and IHC immune profiling data is ongoing to augment translational insights for neoadjuvant chemo/immunotherapies.

http://dx.doi.org/10.1136/jitc-2021-SITC2021.962 